\title{
Enhancement of the reflectivity of Mo/Si multilayer x-ray mirrors by thermal treatment
}

\author{
A. Kloidt, K. Nolting, U. Kleineberg, B. Schmiedeskamp, and U. Heinzmann \\ Universität Bielefeld, Fakultät für Physik, D-4800 Bielefeld 1, Germany \\ P. Müller and M. Kühne \\ Physikalisch-Technische Bundesanstalt, Institut Berlin, D-1000 Berlin 33, Germany
}

(Received 11 December 1990; accepted for publication 14 March 1991)

\begin{abstract}
Thermal treatment of a Mo/Si multilayer stack enhances its reflectivity in the soft $\mathrm{x}$-ray region. The multilayer $x$-ray mirrors are fabricated by electron beam evaporation in ultrahigh vacuum. In situ measurement of the reflectivity during the deposition allows thickness control and an observation of changes in quality of the boundaries. By heating the substrates during deposition we obtain a smoothing of the interfaces. This leads to $\mathrm{x}$-ray mirrors with peak reflectivity around $50 \%$ for normal incident radiation of wavelengths between 130 and $140 \AA$.
\end{abstract}

The Mo/Si multilayer system is a good material combination for $\mathrm{x}$-ray mirrors in the wavelength region of $\lambda=125-250 \AA .{ }^{1}$ To reach a high peak reflectivity near the theoretical limits it is necessary to avoid an increase of roughness throughout the entire stack. For both of the most widely used deposition methods, electron beam evaporation ( $e^{-}$-beam evaporation) and sputtering, the quality of the interfaces depends on the processing and deposition conditions. ${ }^{2,3}$ While different groups found parameters for the sputtering process which yield normal incidence reflectivities around $50 \%$ between 124 and $150 \AA$ (Refs. 2-5) $e^{-}$-beam evaporation yielded considerable lower reflectivities. ${ }^{2,3,6}$ An enhancement of the normal incidence reflectivity by thermal treatment during growth has been reported by Ogura et al. and Niibe et al. ${ }^{2,3}$ but the maximum values obtained for the reflectivity were below $25 \%$. Slaughter et $a .^{7}$ obtained a strong enhancement in the reflectivity for Mo/Si multilayers but with a period which is by a factor of two larger than those in this work and in Refs. 2, 3, and thus by far less sensitive to interfacial imperfections. Smoothing of interfaces in x-ray mirrors can also be obtained by ion polishing ${ }^{8,9}$ but to our knowledge up to now this method has not been applied to the Mo/Si system. In this work we have fabricated Mo/Si multilayers by $e^{-}$-beam evaporation and annealing during growth at temperatures of 150 and $175^{\circ} \mathrm{C}$, because the works ${ }^{2,3,7}$ suggested that at least a local maximum in the smoothing effect should exist in this temperature range. The layer systems were characterized by in situ measurements of the reflectivity of carbon $K$ radiation versus multilayer thickness, as well as by ex situ studies with $\mathrm{Cu} K_{\alpha}$ versus grazing angle and with synchrotron radiation versus wavelength for the complete $\mathrm{x}$-ray mirror.

The deposition is performed in ultrahigh vacuum (base pressure $10^{-10}$ mbar, operational pressure $1-4 \times 10^{-9}$ mbar) with $2 \mathrm{k} \mathrm{W} e^{-}$beam evaporation sources. ${ }^{6}$ The distance between the evaporation sources and the substrates is $65 \mathrm{~cm}$. The system is completed by quartz oscillators for rate and layer thickness monitoring. The fabrication process of the multilayers is controlled by the in situ reflectivity method. ${ }^{10,11}$ The monitor wavelength chosen must give a sufficient signal during growth for both materials. We use $C-K$ radiation $(\lambda=44.7 \AA)$ at an angle of $\alpha=69.5^{\circ}$ with respect to the surface normal of the substrate. This presents geometry and wavelength results in normal-incidence mirrors for the $80-100 \mathrm{eV}$ region. The typical course of the reflectivity during deposition of a $\mathrm{Mo} / \mathrm{Si}$ stack can be followed in Fig. 1. We change the evaporation material from $\mathrm{Mo}$ to $\mathrm{Si}$ at a maximum and from $\mathrm{Si}$ to Mo at the following minimum of the measured reflectivity curve. Thus an alternating layer system is produced. We observe distinct maxima and minima which turn out to be periodic after a few layer pairs. The initial increase in the reflectivity observed during the Si deposition above the maximum in the Mo deposition is in agreement with theoretical calculations (see Ref. 6). After a few layer pairs the average reflectivity shows no further increase; calculations in Ref. 6 show that under ideal conditions, i.e., without any change in interfacial roughness, the average reflectivity remains nearly constant as in Fig. 1(b), caused by absorption and destructive interference of the $C-K$ radiation in the $\mathrm{Mo} / \mathrm{Si}$ multilayer stack. Therefore this part of the curve is a sensitive indication to changes in quality of the boundaries during the deposition. Furthermore this method of in situ measurement of the reflectivity can observe thickness errors, which can be compensated in the following layer.

The $C-K$ reflectivity measured during growth with the substrate at room temperature (RT) is shown in Fig. 1(a). For the 12 deposited layer pairs we obtain the 12 oscillations of the described characteristic form. The measured average reflectivity decreases after a few periods. The decay of the reflectivity curve indicates an accumulation of roughness at the boundaries during the deposition like Ogura et al., Niibe et al., and Slaughter et al. have already described for deposition at RT. This deterioration in quality of the interfaces reduces the reflectivity of the complete $x$-ray mirror. In order to reach a smoothing of the boundaries we expose our substrate to a thermal treatment during the deposition run at $150^{\circ} \mathrm{C}$ and obtain the in situ reflectivity curve in Fig. 1(b). The measured average reflectivity does not decrease and remains nearly constant for 

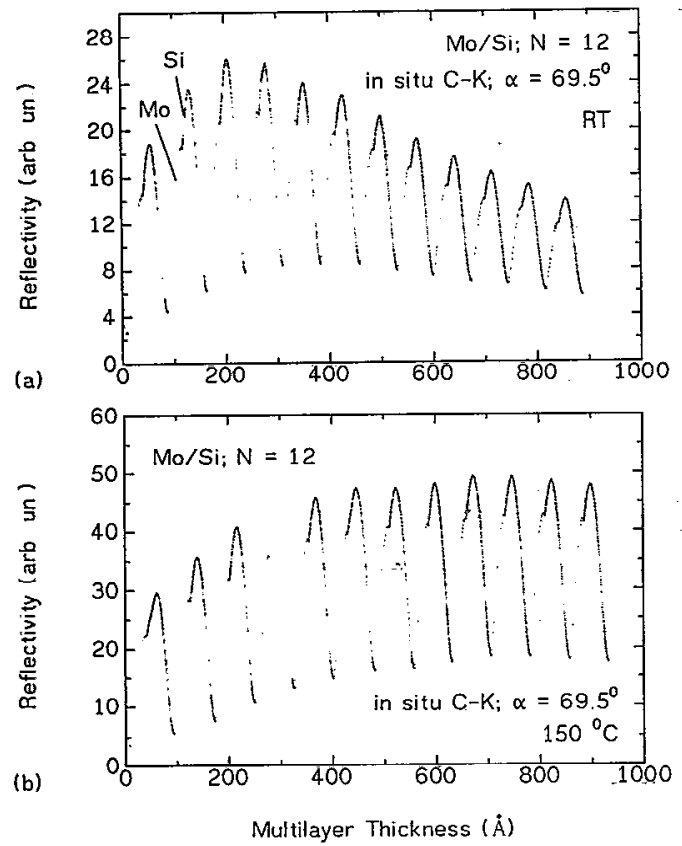

FIG. 1. In situ reflection curve of $\mathrm{Mo} / \mathrm{Si}$ multilayer systems with 12 periods. (a) Substrate at RT, (b) substrate heated during deposition at $150^{\circ} \mathrm{C}$.

the 12 periods. This signifies that the system shows no accumulation of roughness at the interfaces.

First analysis of the optical properties of the completed multilayer has been done by reflectivity measurements with Cu $K_{\alpha}$ radiation $(\lambda=1.54 \AA)$ at grazing incidence. These measurements at short $\mathrm{x}$-ray wavelength were done with a $\vartheta-2 \vartheta$ reflectometer similar to the experiment described by Nevot. ${ }^{12}$ Typical results are reflectivity curves as those displayed in Fig. 2 with a number of Bragg maxima for the first and higher order reflections. As high-order Bragg reflections are by far stronger suppressed by interfacial roughness than the low order Bragg reflections, the occurrence of high-order Bragg reflections indicates the existence of smooth interfaces. Reflectivity curves also give information about thickness ratios of the individual layers in a period of the multilayer. For a quarter wave stack the even order Bragg reflections are suppressed, or in general: the $n$th order Bragg reflection is suppressed if the ratio $\Gamma$ of absorber thickness $d_{\mathrm{Abs}}$ and layer pair thickness $d$ is $1 / n$. Figure 2(a) shows the measured reflectivity curve of the $\mathrm{Mo} / \mathrm{Si}$ multilayer deposited at room temperature, while Fig. 2(b) shows the measurement for the similar system deposited at $150^{\circ} \mathrm{C}$. The measurement of the annealed multilayer shows significantly enhanced reflectivity at the high-order Bragg peaks compared to the reflectivity curve of the nonannealed multilayer. Figure 2(b) exhibits Bragg maxima up to the fifth order, while only three orders can be found in Fig. 2(a). The slower decay of the envelope of the Bragg peaks in the case of the annealed multilayer is significant for reduced roughness of the boundary in the layer stack. Comparing the intensity for the different Bragg orders in Fig. 2 we find a strong suppression of the fourth order and also a reduction of the second Bragg order re-


FIG. 2. (a) Reflectivity measured with $\mathrm{Cu} K_{\alpha}$ radiation at grazing angle $\vartheta$ for the same two mirrors as presented in Fig. 1. (b) Bragg peaks up to the fifth order are clearly observed in case of the annealed substrate.

flection. This is at first sight surprising, since the in situ reflectivity deposition procedure described above yields a $\Gamma$ by far closer to $1 / 3$ than to $1 / 4 .^{6}$ The data can however qualitatively be explained if an interfacial layer exists between Mo and Si. If the condition $2 d_{\mathrm{Mo}}=2 d_{\text {interfacial layer }}=$ $d_{\mathrm{Si}}$ is fulfilled, complete suppression of the fourth and partial suppression of the second-order Bragg reflection is obtained. It requires a thickness of the interfacial layer of $d / 4$, i.e., about $17 \AA$ in agreement with the value reported in Ref. 7. The best agreement of the curve in Fig. 2(b) with a calculation is obtained for a roughness value $\sigma$ of $4.5 \AA$.

The multilayer are designed as normal-incidence mirrors for the soft $\mathrm{x}$-ray region. Thus measuring the reflectivity versus wavelength with synchrotron radiation is the appropriate test of the coatings. The curves in Figs. 3 and 4(b) were measured in the PTB laboratory at BESSY in

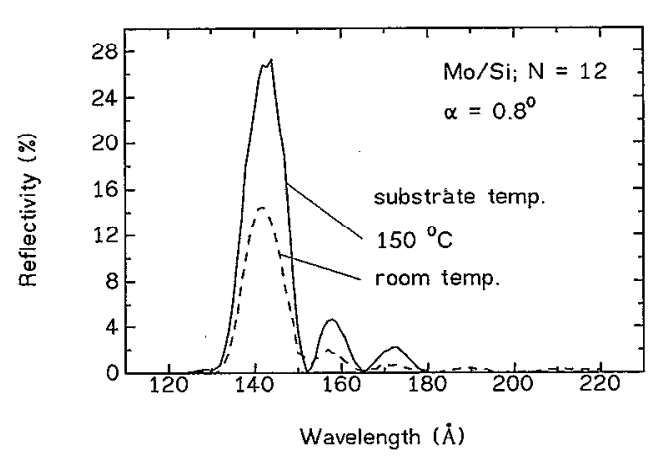

FIG. 3. Reflectivity for synchrotron radiation as a function of wavelength at an angle of $\alpha=0.8^{\circ}$ to the surface normal for the same two mirrors as in Fig. 1. The normal-incidence reflectivity is drastically enhanced by thermal treatment. 

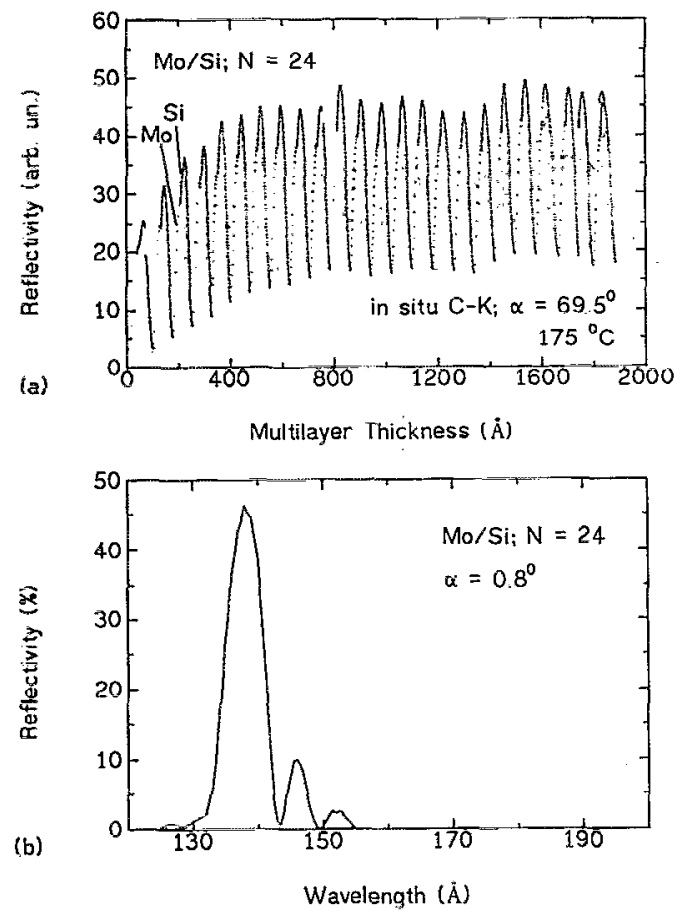

FIG. 4. (a) In situ carbon $K$ reflection curve for 24 layer pairs at $175^{\circ} \mathrm{C}$. After a few periods the average reflectivity remains nearly constant. (b) The reflectivity of the complete $\mathrm{x}$-ray mirror measured by use of synchrotron radiation $R_{\max }=46 \%$ at $138 \AA$.

Berlin. ${ }^{13}$ Figure 3 shows results obtained for two multilayer systems of the same preparation conditions except for the substrate temperature. We observe a remarkable increase of the peak reflectivity by a factor of 2 if we anneal the substrate during growth. For this sample a value of $\sigma=7 \AA$ for the boundary roughness is determined by calculations using the optical constants of Henke et al. ${ }^{14}$ The values obtained from reflectivity at grazing angles and $\lambda=1.54 \AA$ were slightly lower (see above). This difference is probably due to uncertainties of the optical constants in the soft $\mathrm{x}$-ray region. Furthermore we have deposited 24 layer pairs at a temperature of $175^{\circ} \mathrm{C}$. The in situ reflection curve [Fig. 4(a)] shows nearly no change in quality of the interfaces all through the layer pairs and thus promises a high peak reflectivity. This $\mathrm{x}$-ray mirror has a normal incidence peak reflectivity $R_{\max }=46 \%$ at $138 \AA$ [Fig. 4 (b)].

Summarizing, we have fabricated $\mathrm{Mo} / \mathrm{Si}$ multilayer systems by $e^{-}$-beam evaporation. The mirrors were characterized with $\lambda=44.7 \AA$ during the deposition, with $\lambda=1.54 \AA$ at grazing incidence and with synchrotron radiation in the $\lambda=110-220 \AA$ range at normal incidence. We obtain a smoothing of the boundaries by heating the substrate to 150 or $175^{\circ} \mathrm{C}$ during the deposition of the multilayer and a drastic enhancement of the normalincidence reflectivity up to $46 \%$ at $\lambda=138 \AA$. For a multilayer mirror designed for $130 \AA$ A we would thus expect a normal incidence reflectivity of more than $50 \%$ due to the more favorable optical constants. ${ }^{14}$

Special thanks are to $H$. Müller for software development and helpful discussions. Scientific discussion and cooperation with K. F. Heidemann and T. Hellmuth, Fa. Carl Zeiss, Oberkochen, is gratefully acknowledged. The work was financially supported by VDI/ BMFT(FKZ:13N5539).

${ }^{1}$ T. W. Barbee, S. Mrowka, and M. C. Hettrick, Appl. Opt. 24, 883 (1985).

${ }^{2}$ S. Ogura, M. Niibe, Y. Watanabe, M. Hayashida, and T. Iizuka, Proc. SPIE 984, 140 (1988).

${ }^{3}$ M. Niibe, M. Hayashida, and Y. Fukuda, Proc. SPIE 1343, 2 (1990).

${ }^{4}$ J. A. Trail, R. L. Byer, and T. W. Barbee, Appl. Phys. Lett. 52, 269 (1988).

${ }^{5}$ N. M. Ceglio, D. P. Gaines, D. G. Stearns, and A. M. Hawryluk, Opt. Commun. 69, 285 (1989).

${ }^{6}$ B. Schmiedeskamp, B. Heidemann, U. Kleineberg, A. Kloidt, M. Kühne, H. Müller, P. Müller, K. Nolting, and U. Heinzmann, Proc. SPIE 1343, 64 (1990).

${ }^{7}$ J. M. Slaughter, P. A. Kearney, D. W. Schulze, C. M. Falco, C. R. Hills, E. B. Saloman, and R. N. Watts, Proc. SPIE 1343, 73 (1990).

${ }^{8}$ E. Spiller, Appl. Phys. Lett. 54, 2293 (1989).

${ }^{9}$ E. J. Puik, thesis, University of Amsterdam (1990); E. J. Puik, M. J. van der Wiel, H. Zeijlemaker, and J. Verhoeven, Appl. Surf. Sci. 47, 63 (1991).

${ }^{10}$ E. Spiller, A. Segmüller, J. Rife, and R. Haelbich, Appl. Phys. Lett. 37, 1048 (1980).

${ }^{11}$ E. Spiller, Proc. SPIE 563, 367 (1985).

${ }^{12}$ L. Nevot, Acta Electron. 24, 255 (1981/82).

${ }^{13}$ M. Kühne and P. Müller, Proc. SPIE 1140, 220 (1989).

${ }^{14}$ B. L. Henke, E. M. Gullikson, J. C. Davis, M. Fryer, and A. Oren, Proc. SPIE 911, 107 (1988); B. L. Henke, J. C. Davis, E. M. Gullikson, and R. C. C. Perera, Center for X-Ray Optics, Lawrence Berkeley Laboratory (1988), diskette form. 